\title{
LA NANO-OPTIQUE SOUS LA POINTE D'UN MICROSCOPE À EFFET TUNNEL
}

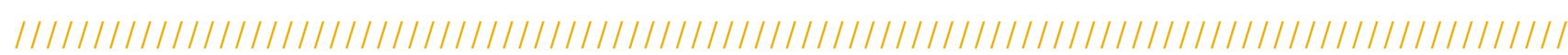

\section{Eric LE MOAL ${ }^{1 ;}$, Elizabeth BOER-DUCHEMIN ${ }^{1, \dagger}$}

${ }^{1}$ Équipe Nanophysique et Surfaces, Institut des Sciences Moléculaires d'Orsay, CNRS - Université Paris-Saclay, 91405 Orsay, France *eric.le-moal@universite-paris-saclay.fr - ${ }^{\dagger}$ elizabeth.boer-duchemin@universite-paris-saclay.fr
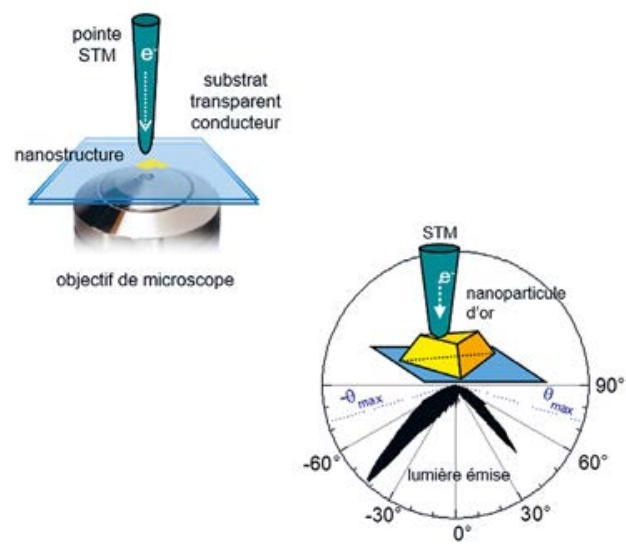

Le microscope à effet tunnel (STM) n'est pas seulement un outil de sciences des surfaces qui produit de magnifiques images résolues à l'échelle atomique. Le courant tunnel sous la pointe du STM est également une source d'excitation optique extrêmement locale, ce qui en fait un extraordinaire outil de la nano-optique. Ici, nous donnons un aperçu des possibilités de cet outil en plasmonique et en électroluminescence, lorsque STM et microscopie optique sont associés dans un même instrument.

Article publié en accès libre sous les conditions définies par la licence Creative Commons Attribution License CC-BY (http://creativecommons.org/licenses/by/4.0), qui autorise sans restrictions l'utilisation, la diffusion, et la reproduction sur quelque support que ce soit, sous réserve de citation correcte de la publication originale.

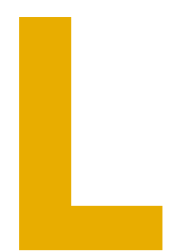

e microscope à effet tunnel (STM) est un fabuleux outil d'imagerie et de spectroscopie électronique. Il permet de sonder la surface des matériaux conducteurs et semi-conducteurs avec une résolution spatiale à l'échelle atomique. Inventé en 1981 [1], cet outil a lancé la révolution des nanosciences [2] et reste incontournable dans ce domaine. Cependant, il est moins connu que le STM est également une nanosource électrique de lumière [3]. Dans ce qui suit, nous présentons l'utilisation du courant tunnel d'un STM comme une nanosonde produisant une excitation optique extrêmement locale, source de plasmons de surface et de photons. En particulier, nous montrons les nouvelles possibilités offertes par la combinaison du STM et de la microscopie optique, pour l'étude des nanostructures plasmoniques et des nouveaux matériaux électroluminescents tels que les monofeuillets de dichalcogénures de métaux de transitions (TMDs).

\section{DE LA LUMIÈRE \\ SOUS LA POINTE DU STM}

Le principe du STM repose sur la mesure d'un courant électronique traversant par effet tunnel l'infime espace $(\approx 1 \mathrm{~nm})$ séparant l'extrémité d'une pointe métallique très fine et la surface d'un échantillon conducteur ou semi-conducteur, entre lesquelles est appliquée une tension de l'ordre du volt. C'est grâce à la mesure de ce courant, qui dépend exponentiellement de la distance pointe-échantillon, que l'image de topographie est formée.

Dès la fin des années 1980, il a été observé que de la lumière est émise par la jonction tunnel durant l'acquisition d'une image STM [4]. Rapidement, cette émission a été attribuée à un processus inélastique, où les électrons traversent la jonction tunnel de telle manière qu'ils perdent une partie de leur énergie, laquelle sert à exciter un mode électromagnétique 


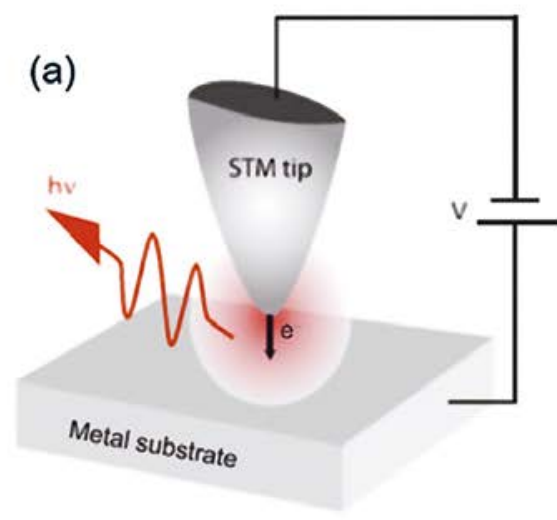

de l'échantillon (voir Fig. 1c). Ce phénomène est notamment responsable de l'excitation de polaritons de plasmons de surface, qui sont des ondes électromagnétiques couplées aux oscillations de la densité électronique à la surface des métaux. Les polaritons de plasmons de surface peuvent être confinés dans des volumes extrêmement petits comparés à la limite de diffraction de la lumière, ce qui motive d'intenses recherches en plasmonique (c'està-dire l'ensemble des technologies qui utilisent ces polaritons comme vecteurs d'information).

\section{COUPLAGE STM - OPTIQUE}

Les premières expériences d'émission sous pointe STM étaient réalisées dans des conditions ne permettant de collecter la lumière que du côté de la pointe (voir Fig. 1a), au moyen d'une fibre optique ou d'une lentille. Ces configurations offrent des efficacités de collection très variables, typiquement de $0,1-1$ $\%$ pour une fibre et $1 \%-10 \%$ avec une lentille. Plus récemment, une configuration bien plus avantageuse en termes de collection a été exploitée, dans laquelle l'émission est détectée en transmission (Fig. 1b) grâce à l'utilisation d'un objectif de microscope à immersion à huile [6] (voir Fig. 2a). Lorsqu'elle peut être mise en œuvre, cette approche permet de collecter plus de $50 \%$ de la lumière émise, jusqu'à $80 \%$ dans
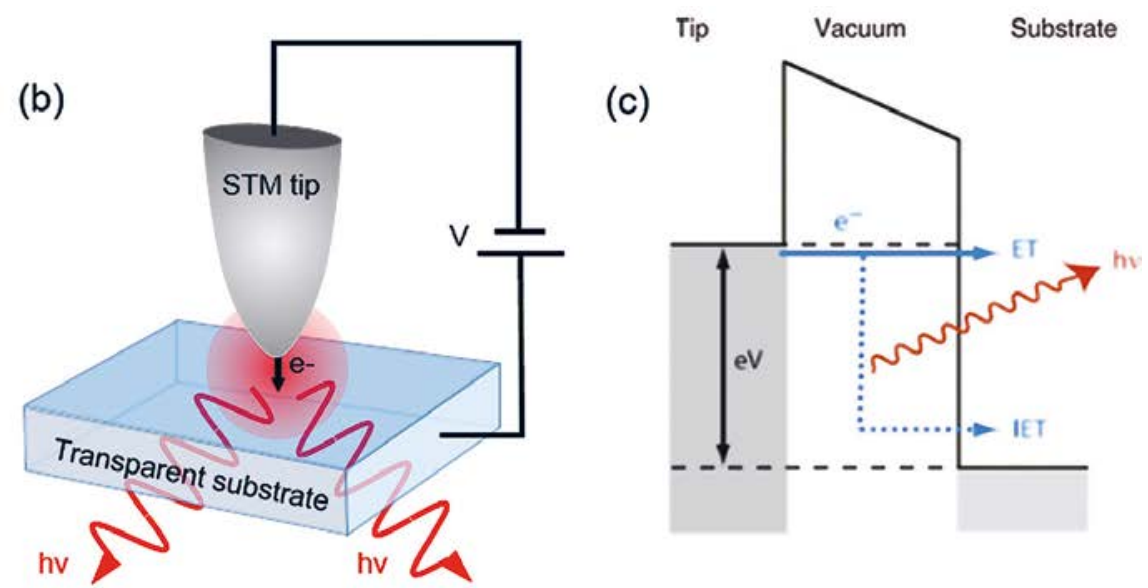

Figure 1.

(a,b) Schémas illustrant l'émission de lumière induite par effet tunnel inélastique dans la jonction d'un microscope à effet tunnel (STM). La lumière émise peut être détectée (a) du côté de la pointe ou (b) à travers l'échantillon. (c) Diagramme d'énergie montrant la jonction pointe-surface polarisée à une tension $V$ et le passage d'un électron par effet tunnel élastique (ET) et inélastique (IET) accompagné de l'émission d'un photon d'énergie hv $\leq$ eV. Adapté de [5] avec la permission d'Elsevier.

Figure 2 .

(a) Schéma d'un microscope optique inversé couplé à un STM. L'échantillon est placé entre la pointe du STM et un objectif de grande ouverture numérique. La lumière émise est collectée en transmission. Les rayons optiques représentés montrent la formation de l'image du plan de Fourier (distribution angulaire). En insert: une image de microscopie électronique à balayage d'une nanoparticule d'or (NP) sur ITO. (b,c) La distribution angulaire de la lumière émise dans le substrat est obtenue à partir des images du plan de Fourier. La direction dans laquelle la lumière est émise dépend de la position latérale de la pointe, car celle-ci détermine quels modes de la NP sont excités. Adapté avec permission de [7]. Copyright (2013) American Chemical Society.
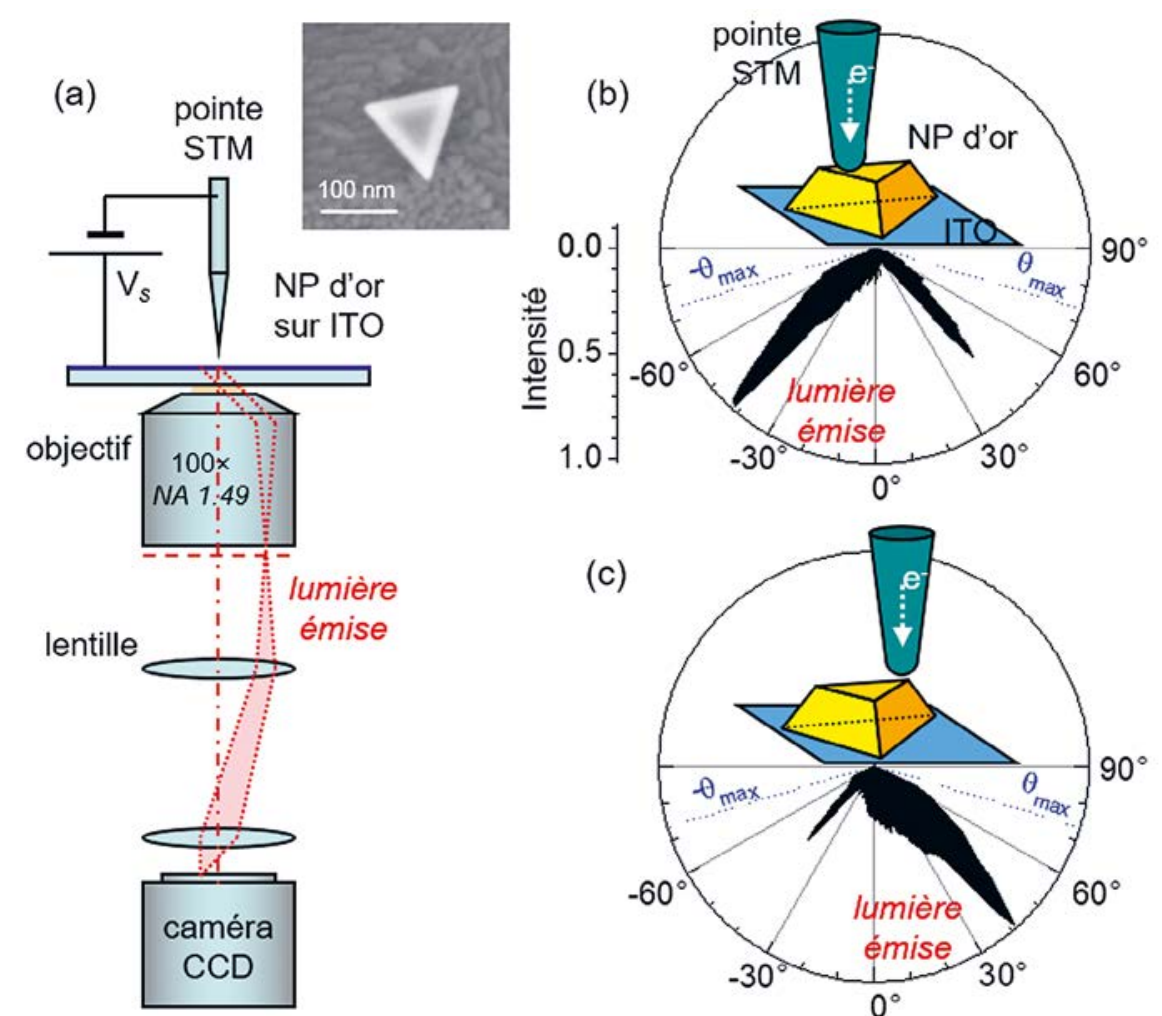

(c)

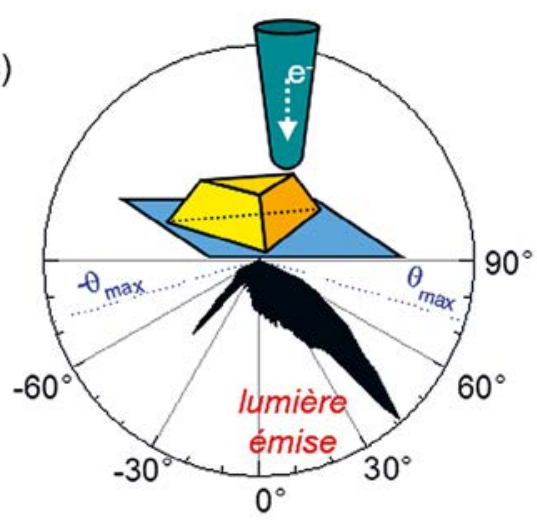


certains cas, du fait que la lumière est majoritairement émise dans le milieu d'indice de réfraction le plus élevé, ici le substrat.

LES ATOUTS DU STM - OPTIQUE L'utilisation du courant tunnel sous la pointe d'un STM comme source d'excitation présente de nombreux avantages par rapport à l'utilisation d'une source de lumière macroscopique. La localisation spatiale de l'excitation par STM permet une sélectivité et une résolution spatiales qui dépassent d'un à plusieurs ordres de grandeur la limite de diffraction de la lumière. L'excitation étant électrique, celle-ci ne produit pas de fond lumineux parasite lié au filtrage imparfait de la lumière d'excitation. De plus, l'excitation par STM est à la fois extrêmement locale et spectralement très large. En effet, l'énergie cédée par les électrons tunnels inélastiques peut prendre toutes les valeurs entre zéro et leur énergie initiale (voir Fig. 1c).

\section{L'association de la microscopie} optique et du STM ouvre de nouvelles possibilités dans l'analyse des émissions de lumière induites par effet tunnel inélastique. En effet, si le courant tunnel permet d'exciter très localement les modes de l'échantillon, le STM ne permet pas de visualiser où a lieu l'émission de lumière, ni dans quelle direction celle-ci a lieu.

\section{LES POSSIBILITÉS \\ NOUVELLES APPORTÉES PAR LA MICROSCOPIE OPTIQUE \\ L'association de la microscopie optique et du STM ouvre de nouvelles possibi- lités dans l'analyse des émissions de lumière induites par effet tunnel iné- lastique. En effet, si le courant tunnel}

permet d'exciter très localement les modes de l'échantillon, le STM ne permet pas de visualiser où a lieu l'émission de lumière, ni dans quelle direction celle-ci a lieu. Ces informations sont pourtant cruciales pour déterminer la nature des modes excités, en particulier si ceux-ci sont des modes propagatifs [6]. L'utilisation d'un objectif de microscope pour collecter la lumière émise en transmission permet de réaliser une image du plan objet, révélant la distribution spatiale de la lumière émise (image dans l'espace réel). De plus, une image du plan focal arrière de lobjectif révèle la distribution angulaire de l'émission (image dans l'espace de Fourier, voir Fig. 2) [7], pouvant être reliée à la relation de dispersion entre énergie et quantité de mouvement des modes propagatifs excités [8]. Enfin, optimiser l'efficacité de collection dans ce type d'expériences est crucial, en raison du très faible flux de photons émis. En effet, l'efficacité quantique des effets tunnels inélastiques est très basse, de lordre de $10^{-6}$ à $10^{-4}$ photons émis par

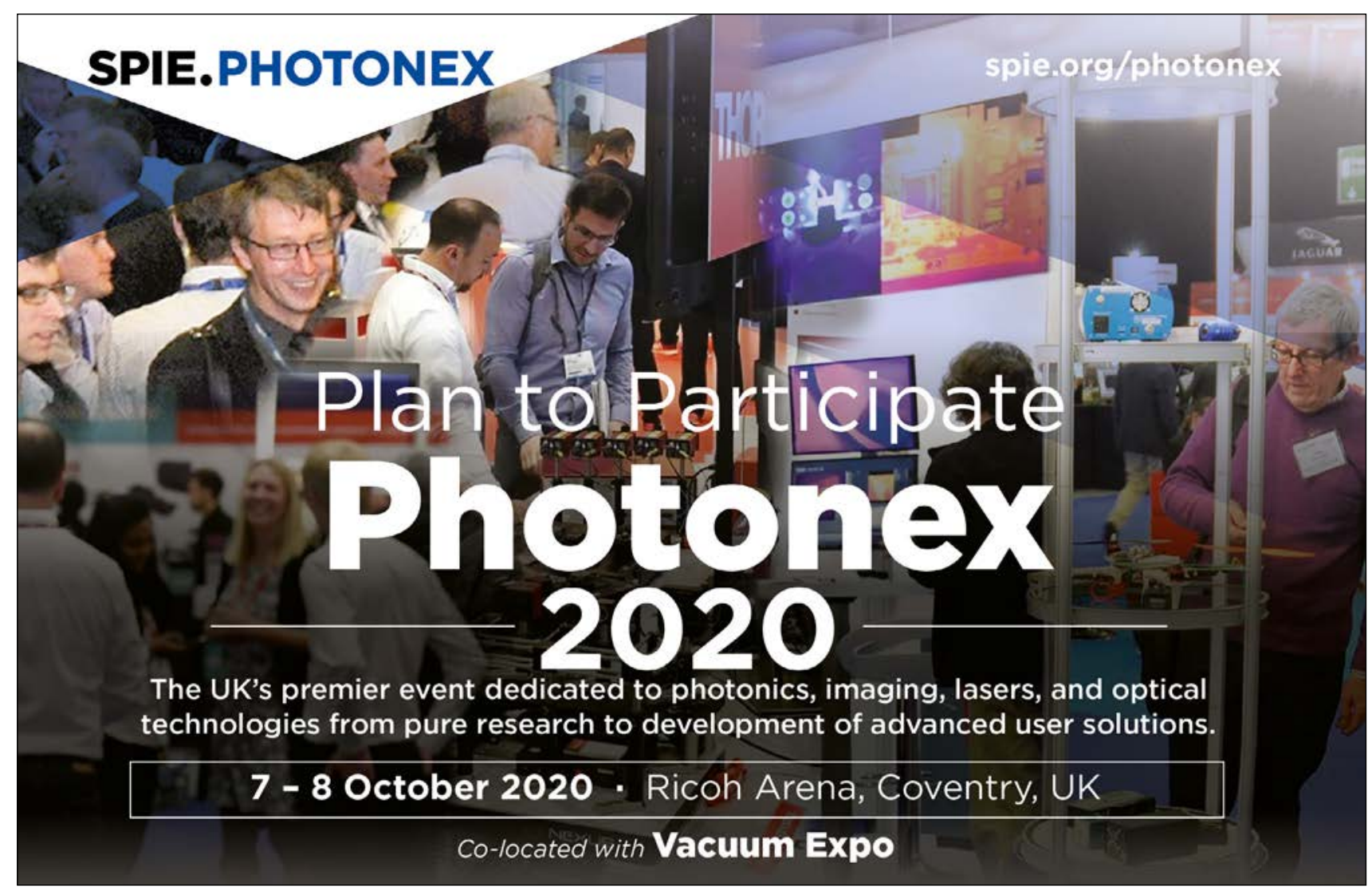


électron tunnel, et les courants utilisés sont faibles (nanoampères).

\section{QUELQUES EXEMPLES D'APPLICATIONS \\ À LA PLASMONIQUE \\ ET L'ÉLECTROLUMINESCENCE}

La figure 2 montre une expérience où les différents modes électromagnétiques d'une nanoparticule (NP) d'or sont excités sélectivement, en contrôlant la position latérale de la pointe sur la NP [7]. C'est grâce à la distribution angulaire de la lumière émise, mesurée dans l'espace de Fourier, que ceci est démontré (voir Figs. $2 b$ et 2c). Par la même occasion, cette expérience démontre le principe d'une nanosource de lumière dont la direction d'émission est contrôlable.

Plus récemment, nous avons utilisé ce même instrument pour cartographier localement les propriétés d'électroluminescence d'un semi-conducteur bidimensionnel, à savoir un monofeuillet de diséléniure de molybdène $\left(\mathrm{MoSe}_{2}\right)$ [9]. La figure $3 \mathrm{~b}$ montre une image de microscopie optique dans l'espace réel enregistrée pendant l'excitation par STM. Cette image révèle que des excitons (ou paires d'un électron et d'un trou liés) générés sous la pointe du STM peuvent diffuser sur plusieurs micromètres avant leur recombinaison radiative. La cartographie de luminescence présentée en figure 3, dont la résolution spatiale est estimée à $10 \mathrm{~nm}$ environ, révèle les effets de la topographie locale du monofeuillet de $\mathrm{MoSe}_{2}$ (Fig. 3c) sur l'efficacité quantique d'électroluminescence (Fig. 3d). Ceci permet de mieux comprendre et contrôler les performances de dispositifs optoélectroniques basés sur ce type de semi-conducteurs.

\section{CONCLUSION}

Le STM n'est pas uniquement un outil d'imagerie et de spectroscopie électronique. C'est également une source d'excitation optique qui peut générer localement des photons, des polaritons de plasmons de surface et des excitons. Coupler le STM à un microscope (a)

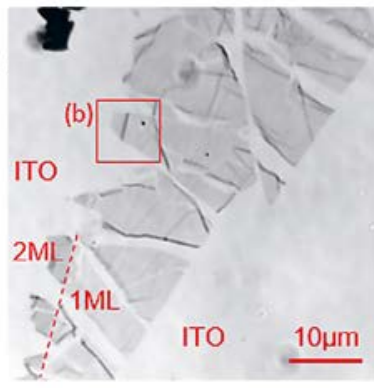

(b)

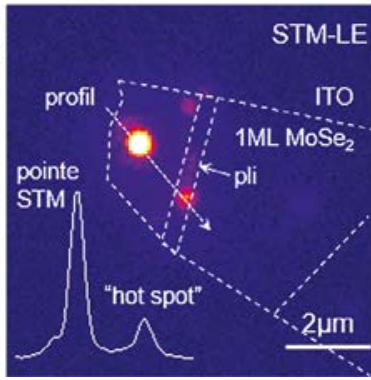

(c)

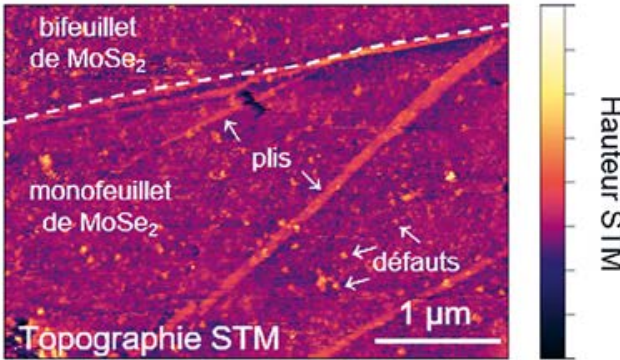

(d)

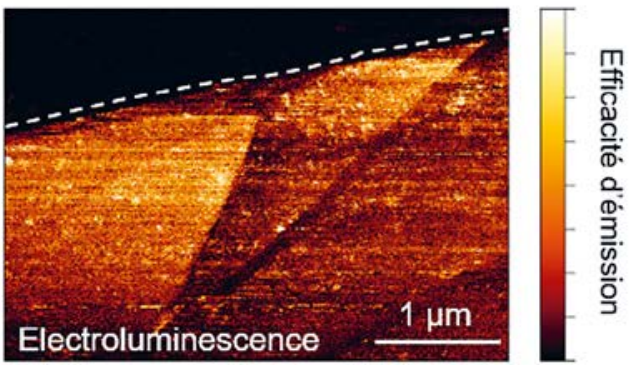

Figure 3.

Mesures d'électroluminescence locale d'un TMD, réalisées avec un STM couplé à un microscope optique (voir schéma en Fig. 2a) [9]. (a) Image optique en lumière blanche d'un feuillet de $\mathrm{MoSe}_{2}$. (b) Image dans l'espace réel de la luminescence induite pendant l'excitation par STM (région encadrée en a). La présence de pics d'émission (hot spots) distants de la pointe révèle que des excitons créés sous la pointe diffusent sur plusieurs micromètres avant leur recombinaison radiative. (c) Topographie STM et (d) cartographie de luminescence mesurées simultanément, révélant les effets de la géométrie locale du feuillet sur l'efficacité quantique (nombre de photons émis par électron tunnel) d'électroluminescence. Le changement de contraste abrupte au milieu de la cartographie de luminescence révèle un joint de grain quasiment indétectable sur la topographie. Figures adaptées avec permission de [9]. Copyright (2019) by the American Physical Society.

optique permet de détecter efficacementlesémissions de lumière induites par le courant tunnel et de résoudre leur distribution spatiale, angulaire et spectrale. Ces expériences trouvent des applications dans des domaines tels que la plasmonique, pour tester et optimiser des microcomposants optiques destinés à un circuit plasmonique, ou loptoélectronique, pour cartographier à léchelle nanométrique les propriétés excitoniques de semi-conducteurs destinés à composer des microdispositifs électroluminescents. Les perspectives de développement de cette technique incluent notamment la résolution temporelle par le déclenchement optique du courant tunnel et l'extension vers des domaines de fréquences optiques plus basses (infrarouge moyen) pour létude des polaritons de phonons.

RÉFÉRENCES

[1] G. Binnig et al., Phys. Rev. Lett. 49, 57 (1982)

[2] M. F. Crommie et al., Science 262, $218(1993)$

[3] K. Kuhnke et al., Chem. Rev. 117, 5174 (2017)

[4] J. K. Gimzewski et al., EPL 8, 435 (1989)

[5] F. Rossel et al., Surf. Sci. Rep. 65, $129(2010)$

[6] T. Wang et al., Nanotechnology 22, $175201(2011)$

[7] E. Le Moal et al., Nano Lett. 13, 4198 (2013) [8] S. Cao et al., Opt. Express 27, 33011 (2019) [9] D. Pommier et al., Phys. Rev. Lett. 123, $027402(2019)$ 